\title{
Capabilities of processing and visualization of production facilities diagnostic data
}

\author{
Matej Kandera ${ }^{1, *}$, Miroslav Císar ${ }^{1}$, Ivan Zajačko, \\ ${ }^{1}$ University of Žilina, Faculty of Mechanical Engineering, Department of Automation and Production \\ Systems, Univerzitná 1, 01026 Žilina, Slovakia.
}

\begin{abstract}
One of the main requirements of the safe and sustained workflow of production facilities and machines, is the proper choice and implementation of suitable technical diagnostic method. In the broader sense technical diagnostics includes approaches and methods of technical objects condition determination. This process is based on specific devices parameters inspecting, which values are directly or indirectly caused by certain changes of monitored production facilities condition. In order to reliably detect faults in right time, selected parameters indicating status of the production device should be monitored continuously. At a time when an Internet of Things platforms implemented to the industry business systems is the great temptation from the view of investors and company prestige are these principles common in the process of machine parameters online monitoring and its cross connections within the business realms. This paper describes specific software ThingWorx, which is a great example of sophisticated and universal IoT platform and also describes its application for production facilities diagnostic data collection and visualization.
\end{abstract}

Keywords: condition monitoring, maintenance, Thing

\section{Introduction}

High manufacturing quality, reliability, product accuracy and last but not least efficient use of financial resources, are the main parameters which would define a successful engineering company. Since at the time when the great effort is oriented on this parameters, like high quality combined with economic efficiency of manufacturing process, there is limited space for accidental faults of production from the manufacturers side which can immediately cause profit decrease and even under certain circumstances can cause the regression of the whole company. Downtimes of the manufacturing or assembling processes caused by unexpected faults of all types represent the most significant issues mainly in the fully automated production lines, where literally every tenth of second play its role. Especially in automotive industry where even a few seconds lasting downtime can cause significant financial losses. For these and many other reasons, the technical diagnostics and thus proper and effective planning of maintenance of production facilities are of great importance. [1]

On the other hand, diagnostics of the cause of faults or damage and subsequently production machine and facilities maintenance represents a significantly demanding financial

\footnotetext{
* Corresponding author: matej. kendra@fstroj.uniza.sk

Reviewers: Nicoale Ungureanu, Jozef Jurko
} 
part within the business costs, mainly considering smaller or starting companies. Not to mention cases, when facility damage or degradation is detected after machine breakdown or when damage became unsustainable. From these and many other reasons, it is required to reveal previously mentioned faults and degradations and its progress as soon as possible to ensure minimum financial repairs impact. In the numerous number of fault cases, choice and application of suitable diagnostic method can be the right solution. Choice of such a system is dependent on a type of faults and parameters that we want to detect and also on the type of the machine or device on which we want to apply such a diagnostic method, whether it will be implemented to some sort of assembly or production line, CNC machines, industrial robots or other comparable devices or technical objects. Selection of the right choice also depends on the quantity of the diagnosed devices and on special requirements, for example "machine to machine communication". [2,3]

Real-time monitoring systems and devices are often implemented for this particular purpose. This systems, also known as condition based monitoring systems are capable of online information reflecting of all important machine parameters. Condition monitoring is based on non-destructive and non-invasive methods of measuring of required system characteristics such as frequencies or modal domains measuring and verification for the purpose of detecting whatever deviations. These shall indicate machine failure or degradation of any device parts in advance. In the field of mechanical engineering these monitoring systems have to increase economical profitability and to simplify production facilities maintenance in order to justify their implementation.

This article deals with maintenance, with the aim focused mainly on area of the condition monitoring. There are few platforms chosen as an example of such a software for developing web applications which can process and visualize diagnostic data acquired from diagnosed devices or facilities and subsequently implement this devices, to the Internet of Things. The most advantageous candidate is the use of a large PTC tool called ThingWorx. It is presented as an Internet of Things platform that allows to easily create web applications for the collecting, processing and visualization of data obtained from the sensory and control subsystems of various production facilities. Main purpose is to use the above-mentioned software to capture and monitor data defining the machine state during production and also take advantages of the tools and services that the software provides to the user.

\section{Condition based maintenance}

In order to understand why the use of condition monitoring is important or relevant, it is necessary to understand what is actually the essence of condition-based maintenance and what is the difference between the concepts of technical diagnostics and machine condition monitoring.

According to the CSN EN 60300-3-11 standard, maintenance is defined as a set of all technical, administrative and managerial steps or actions during the lifecycle of the machine on which the maintenance is aimed to sustain its serviceability or to return it to the maintained state. [4]

The most common types and methods of technical maintenance are listed in the Figure 1. Important for us now is a part of "before detected faults" which contains bigger group of preventive maintenance and specifically we are dealing with condition based maintenance. This method is also known as predictive maintenance, because the potential failure of the machine is predicted by regular condition monitoring of chosen parameters, that indicates the condition of the equipment, which is measured continuously or periodically. Following maintenance is therefore performed at the optimal time. Benefits of the predictive maintenance are obvious in comparison to methods like "run-to-brake" or preventive maintenance, but it requires access to the reliable condition monitoring techniques which can 
not only define or describe the current state but also provide reasonable prediction for the remaining lifetime. [5,6]

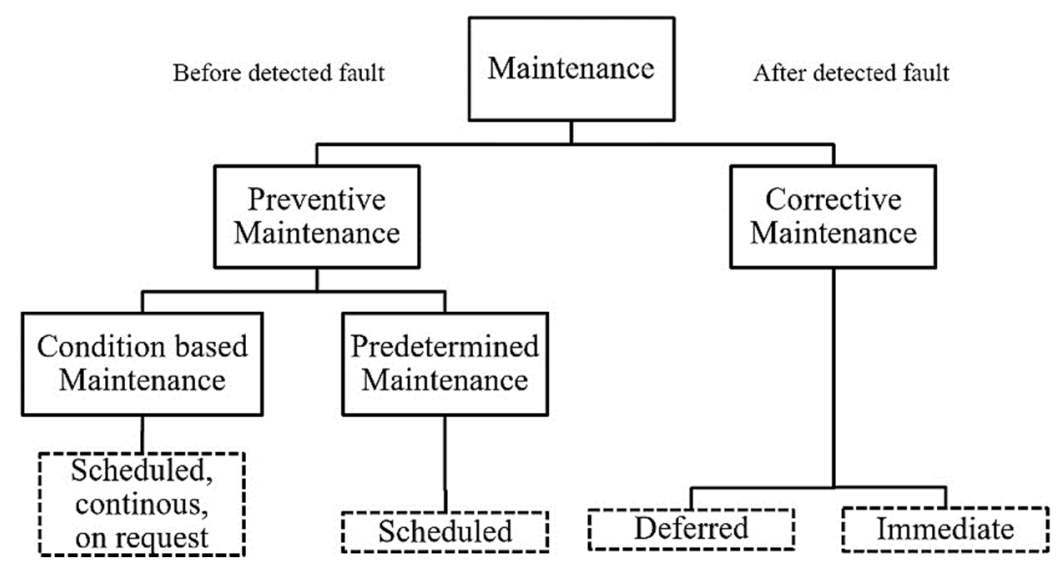

Fig. 1. Maintenance approaches and strategies.

\section{Condition monitoring}

Condition monitoring does not serve to reveal the reason of fault. It should serve only to detect the fact that there are some undesirable deviations in the monitored values or that some kind of fault has occurred. This process is based on tracking chosen parameter values and their changes and is a major component of predictive maintenance. Main aim of condition monitoring relies on the ability of monitoring present state or condition of devices and on ability of predicting of monitored parameters to the future while the device is in operation. This means that information about internal effects must be obtained externally while the machines are in operation. [6,7]

Machine condition monitoring is an important part of condition-based maintenance $(\mathrm{CBM})$ that is recognized as the most effective maintenance strategy in a wide range of industries. Before preferred "run-to-break" strategy has ensured maximum operational time between shutdowns, but the breakdowns during using this strategy have been occasionally catastrophic, with serious security consequences, production losses, and cost of subsequent repairs. The first response to the deficiencies of this strategy was "preventive maintenance", in which device is repaired at certain intervals, thus ensuring very little possibility of breakdowns between these repairs. However, the disadvantage of such a maintenance strategy is that it is in some cases carried out even though it is still unnecessary, large number of spare parts and, last but not least, a lot of repair time are consumed, which reflects on its financial demands. Condition monitoring can improve Overall Equipment Effectiveness (OEE) with actionable insights and alerts at every level of production lines. [6]

Whole process begins on the production line, where sensors of different types can be installed as required or as are requested by the customer. The most commonly monitored parameters are vibrations, temperature, lubricant degradation or contamination, and electric current. Data from the sensors is processed in the data connectivity gateway to which they are connected either directly by Ethernet, serial ports like RS-232 or are connected via Wi$\mathrm{Fi}$, Bluetooth or another wireless method, where the data is translated between sensor protocols and processed before upcoming procedure. Subsequently data is processed in cloud services, for example using a specialized IoT platform. Then virtual representation is created, with identical properties like real monitored object which represents digital a reflection of 
the monitored device and on this twin monitored properties and values are represented. The measured data is in the end visualized by various visual elements in the web based user interface or data can be further exported. Another solution is the setting of limits after which the system send a warning to the specific mail or otherwise notify the responsible person. Structure of such a condition monitoring system is described on the figure 2.

Production line

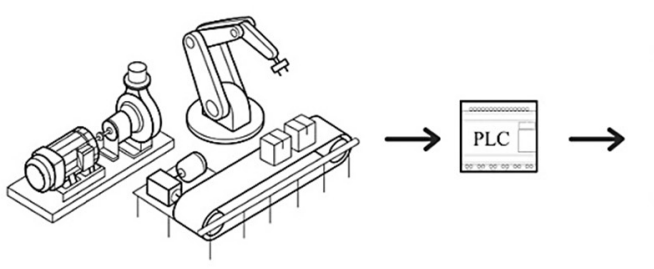

Data Cloud Connectivity Services Gateway
Digital Twin

dashboards and services

Fig. 2. Structure of condition monitoring system

\section{Example of application}

There are many ways for the purpose of creating application suitable for condition monitoring. One of them is to use independent programs for each part of process from data acquiring thru data processing to the form in which it is possible to analyse them until the last step which is building and compiling specific program in one of the commonly used programming languages and creating user interface for the purpose of graphical representation of monitored parameters values. [8]

Another way to deploy an application for condition monitoring is the possibility of using one of the big platforms. These can be strictly specialized only on condition monitoring or can offer solutions for predictive maintenance too. In these days is big trend to associate industry with the phenomenon of upcoming Industry 4.0 and because of this reason there cannot be omitted platforms that make it possible to implement Internet of Things solutions. Their tools can be also used to create web-based applications for condition monitoring and event monitoring of the single machine or whole workshop machinery. Among the best rated IoT platforms of the year 2018 are included for example: Amazon Web Services, Microsoft Azure IoT, Google Cloud Platform and one of the best rated platforms is also ThingWorx from PTC. All of these software serves to M2M (machine to machine) connection, respectively for the interconnection of individual devices within the enterprise and offer the possibility of visualization of the measured parameters which are in our case, diagnostic data from the production machines or production lines.

However, significant level of programming skill is required from the user or programmer to implement most of these platforms. ThingWorx is in this aspect more user-friendly as it allows to develop IoT web-based applications without considerable large knowledge of programming. Instead it works on intuitive "drag and drop" method and uses pre-created objects, so called widgets and things.

\subsection{ThingWorx}

This particular software allows users to build a specific and unique web applications from defining a digital representation of examined object thru defining properties and services to building its final visual interface. The ThingWorx Industrial IoT Platform delivers tools and 
technologies that allow to rapidly develop and deploy powerful IoT applications and AR experiences too. ThingWorx is a complex of entities which represent real processes, solutions and applications with which it is possible to create or model real solutions of specific tasks in a way based on creation of digital twins. Digital twin in this scope of view represents exact reproduction of certain physical object or real system across its lifecycle using a real-time data for purpose of understanding, learning and reasoning. [9,10]

As a description can indicate, ThingWorx platform is based on creation of the "Things". Thing is the digital representation of real world subjects or processes with its own properties and business logic. Structure of such a "Thing" is described in the figure 3., where are four main characteristics shown.

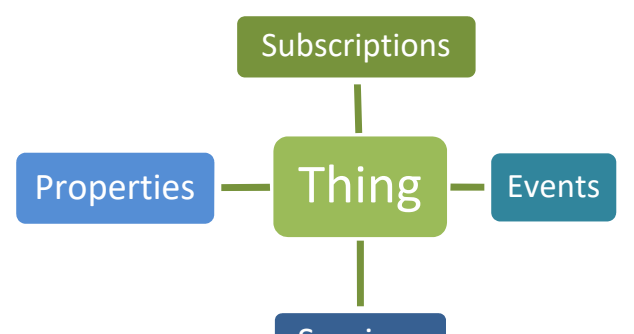

Services

Fig. 3. Structure of the ,Thing “.

Main characteristics of the Thing are:

- Properties - also can be thought as parameters or variables. Represents behaviour of actual thing or just modelled process.

- Services - every function which is ThingWorx allowed to execute. They can be defined at the level of creating a ThingTemplate, ThingShape or Thing.

- Events - represents change of the state or in value of some property. Can interrupts ThingWorx Core and subscribe to for purposes of receiving notification about errors or some faults.

- Subscriptions - it is name for each action which is executed as a stimulus when one of the defined events occurs.

Values of all these characteristics are configured by application programmer and are bound to the created thing. [9]

\subsection{Application development example}

As mentioned in chapter above, the basis of work in this software is to define "Things" and their characteristics. That is why the development of the application in this platform starts with this part. It is not a strict steps sequence ordered during the application development process but there are some main steps that user will not avoid.

One of these steps is defining or creating of the Tags, which serves to organize the work for the purpose of easiest saving and exporting process, categorizing and grouping individual items throughout platform, version control and for the purpose of searching and migrating entities between serves. In general Tag consists of two parts: Vocabulary and term (vocabulary: term) and it is recommended to create tags just before creating other entities in this platform. [9]

It is recommended to take advantage of defining a ThingTemplate during the process of creating individual Things. ThingTemplates are reusable building blocks used to maintain 
scalability and flexibility of our application development. By using ThingTemplates, it is possible to define a set of similar or the same objects with those of the defined basic properties and services. One ThingTemplate is therefore capable of serving as a multi-item template without the need for repetitive and time-consuming multiple submissions of the same data to each Thing. Within the ThingTemplate creation, there is a defining of properties included, that can be static or dynamic, persistent, and can be logged. Subsequently during creating Thing, the process is followed by the user filling a name that would clearly defines what device is it representing and bind the new thing with the pre-created ThingTemplate and assigns the required tags to it. Entering all of this data is done through a relatively simple tables filling with additional Java editing options. $[8,10]$

For the purpose of data storing there might be used Value Stream. This tool represents one of the way, quick and easy, to record the values or data with timestamp in Foundation Server. Other possibilities are to use data tables or simply the streams. When creating the application for condition monitoring in ThingWorx, one of the most important parts is the definition of a Data source that represents real-time data values. Data acquired from the sensors on machines or another device, can be securely extracted to the ThingWorx using OPC UA (Open Platform Communications Unified Architecture), or using preferred industrial data connectivity protocol. Data can be using OPC server communicated continuously among PLCs on the shop floor, RTUs (Remote Terminal Units) in the field, Human Machine Interface (HMI) stations, and software applications on PCs desktops. Main benefit of OPC Compliance usage is, that it allows real-time communication even between hardware and software from different vendors. For the purpose of this demonstration, there was an OPC software KEPServerEX 6 used. Other options for ThingWorx connectivity is usage of ThingWorx Edge SDKs, Edge Microserver, REST API or Device Clouds (AWS IoT, Azure IoT Hub, ...) while each of these options has its pros and cons. [9,11]

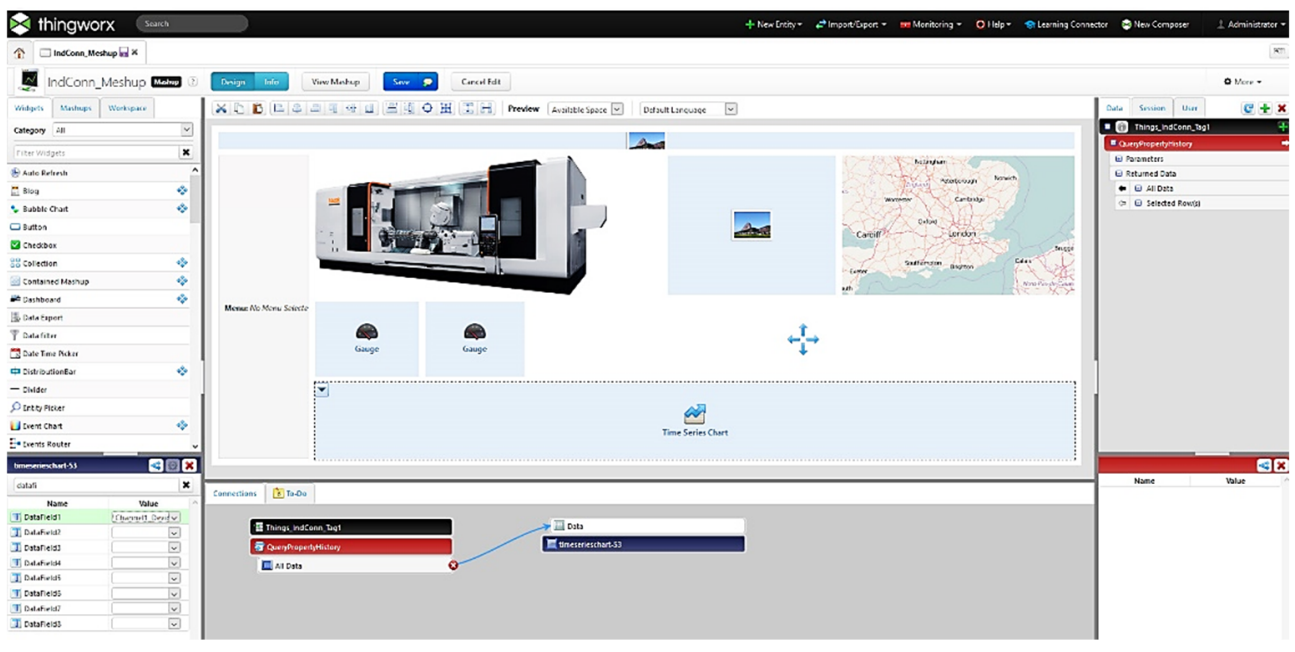

Fig. 4. Mashup builder user interface.

Figure 4 illustrates an example of the Mashup builder interface, the creating module for the application final user interface. Mentioned interface creation is based on a very intuitive "drag-and-drop" system using various widgets such as maps, spreadsheets, charts, various types of buttons and gauges, and various other elements. Platform offers the option to add 
other required widgets that are not found in the main menu via the marketplace. The data for each widget can be bound from the Data Table again with drag-and-drop method. [9]

Figure 5 shows the appearance of the proposed user environment as seen by the users in their browser.

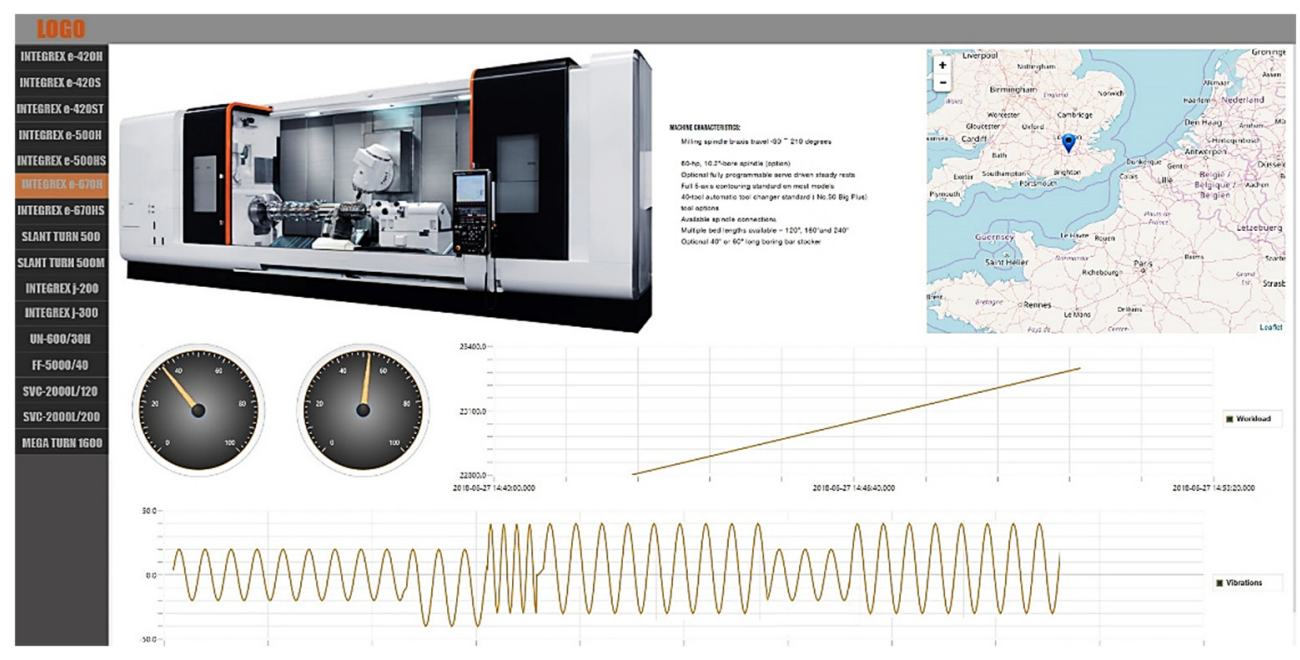

Fig. 5. Example of the final user interface created in "Mashup builder".

\section{Conclusion}

Machine condition monitoring as an important part of condition based maintenance, which is more and more recognized as the most efficient strategy for carrying out maintenance in industry. The benefits of such monitoring can be summarized in several parts. First of all, it allows to implement predictive maintenance as it needs actual data about the values of the machine parameters in this maintenance method. The condition monitoring also accelerates the detection of defects or wear of machine parts that would otherwise be detected only after detailed measurements or during the machine maintenance. As in the case of human diseases, even in the case of industrial facilities, it is true that the sooner these defects or deviations from the normal values are detected, the less expensive and easier it is to remove them and return the machine to its fully functional state. CM also ensures safe operation and preserving constant machine accuracy without undue deviations and quality of manufactured parts. Another benefit could be real-time online representation and visualization of measured parameter values. Such representation is understandable, intuitive, and applicable even without the necessity for special training.

The platform presented in this article offers a solution for the implementation of condition monitoring and the creation of web based monitoring applications based on digital representations of real objects that have their own properties and business logic. The main advantage of such solution, is that it allows to create condition monitoring applications in a simpler way, in comparison to other platforms as it works mainly on drag-and-drop principle, it offers large amount of ready to use objects and templates, and it does not require knowledge of high-level programming languages.

The outputs of paper was supported by grant VEGA 1/0504/17 "Research a development of methods for multicriterion accuracy diagnostic of CNC machines. 


\section{References}

1. V. Bulej, J. Uríček, V. Poppeová, R. Zahoranský, M. Rupiková, Study of the workspace of hybrid mechanism Trivariant. Applied mechanics and materials. ISSN 1660-9336, 436, 366-373 (2013)

2. I. Kuric, V. Bulej, M. Sága, P. Pokorný, Development of simulation software for mobile robot path planning within multilayer map system based on metric and topological maps. International journal of advanced robotic systems, 14, (2017)

3. V. Bulej, G. V. Soianovici, V. Poppeova, Material flow improvement automated assembly lines using Lean logistics. Annals of DAAAM for 2011 \& Proceedings of the 22nd international DAAAM symposium "Intelligent manufacturing \& automation: Power of knowledge and creativity". ISBN 978-3-901509-83-4, 253-254 (2011)

4. H. Opocenska, M. Hammer. Contribution to maintenance issues in company practice. MM Science Journal, 12, 748-755 (2015)

5. V. Pet'ková, Teória a aplikácia vybraných metód technickej diagnostiky. 1. publication. Košice : Technická univerzita, 235 p., ISBN 978-80-553-0483-0. (2010)

6. R., B. Randall, Vibration-based Condition Monitoring: Industrial, Aerospace and Automotive Applications. Chicester: John Wiley \& Sons, Inc., 308 p. ISBN: 978-0-47074785-8 (2011)

7. I. Kuric, I. Zajačko, M. Císar, Analytical intelligence tools for multicriterial diagnostics of CNC machines. Advances in Science and Technology Research Journal, 10.32., (2016)

8. J. Stanček, V. Bulej, Design of driving system for scissor lifting mechanism. Academic journal of manufacturing engineering, ISSN 1583-7904, 13, 38-43 (2015)

9. ThingWorx Glossary. [online] Available on Internet: https://developer.thingworx.com /resources/guides/thing worx- glossary/glossary

10. E. Pivarčiová, et al., Analysis of control and correction options of mobile robot trajectory by an inertial navigation system. International Journal of Advanced Robotic Systems, 15.1: 1729881418755165., (2018)

11. B. Mičieta, M. Edl, M. Krajčovič, L'. Dulina, P. Bubeník, L. Durica, V. Biňasová, Delegate MASs for Coordination and Control of One-Directional AGV Systems: A Proof-of-Concept. International journal of advanced manufacturing technology, 94, 415431. ISSN 0268-3768 (2018) 